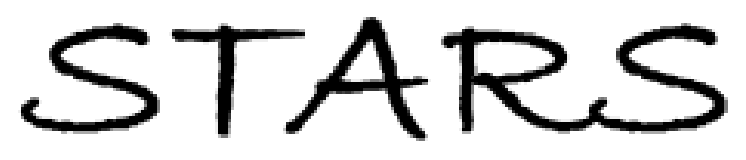

University of Central Florida

STARS

$1-1-2007$

\title{
Wide-view transflective liquid crystal display for mobile applications
}

Hyang Yul Kim

University of Central Florida

Zhibing Ge

University of Central Florida

Shin-Tson Wu

University of Central Florida

Seung Hee Lee

Find similar works at: https://stars.library.ucf.edu/facultybib2000

University of Central Florida Libraries http://library.ucf.edu

This Article is brought to you for free and open access by the Faculty Bibliography at STARS. It has been accepted for inclusion in Faculty Bibliography 2000 s by an authorized administrator of STARS. For more information, please contact STARS@ucf.edu.

\section{Recommended Citation}

Kim, Hyang Yul; Ge, Zhibing; Wu, Shin-Tson; and Lee, Seung Hee, "Wide-view transflective liquid crystal display for mobile applications" (2007). Faculty Bibliography 2000s. 7297.

https://stars.library.ucf.edu/facultybib2000/7297

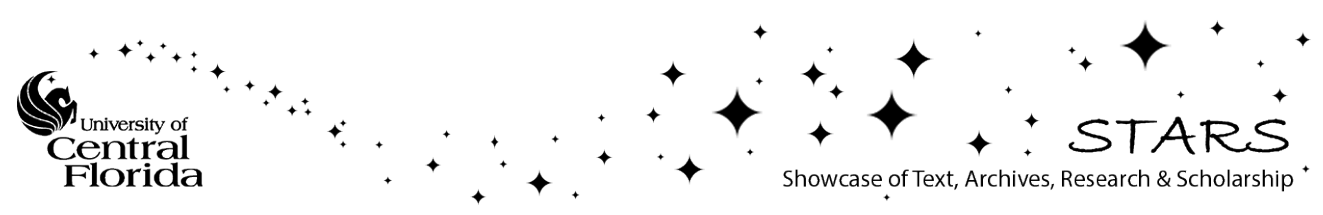




\section{Wide-view transflective liquid crystal display for mobile applications}

Cite as: Appl. Phys. Lett. 91, 231108 (2007); https://doi.org/10.1063/1.2822410

Submitted: 02 November 2007 . Accepted: 16 November 2007 . Published Online: 05 December 2007

Hyang Yul Kim, Zhibing Ge, Shin-Tson Wu, and Seung Hee Lee

\section{ARTICLES YOU MAY BE INTERESTED IN}

Cholesteric liquid crystal/polymer dispersion for haze-free light shutters

Applied Physics Letters 60, 3102 (1992); https://doi.org/10.1063/1.106765

Electro-optic characteristics and switching principle of a nematic liquid crystal cell controlled by fringe-field switching

Applied Physics Letters 73, 2881 (1998); https://doi.org/10.1063/1.122617

Low voltage blue-phase liquid crystal displays

Applied Physics Letters 95, 231101 (2009); https://doi.org/10.1063/1.3271771
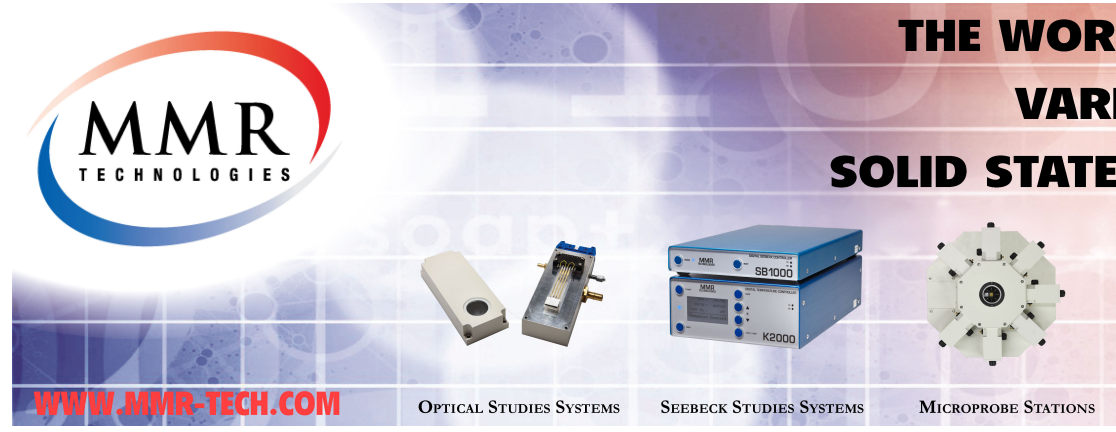

THE WORLD'S RESOURCE FOR VARIABLE TEMPERATURE 


\title{
Wide-view transflective liquid crystal display for mobile applications
}

\author{
Hyang Yul Kim, Zhibing Ge, and Shin-Tson Wu ${ }^{a}$ \\ College of Optics and Photonics, University of Central Florida, Orlando, Florida 32816, USA \\ Seung Hee Lee \\ Polymer BIN Fusion Research Center, School of Advanced Materials Engineering, \\ Chonbuk National University, Chonju, Chonbuk 561-756, Korea
}

(Received 2 November 2007; accepted 16 November 2007; published online 5 December 2007)

\begin{abstract}
A high optical efficiency and wide-view transflective liquid crystal display based on fringe-field switching structure is proposed. The transmissive part has a homogenous liquid crystal (LC) alignment and is driven by a fringe electric field, which exhibits excellent electro-optic characteristics. The reflective part has a hybrid LC alignment with quarter-wave phase retardation and is also driven by a fringe electric field. Consequently, the transmissive and reflective parts have similar gamma curves. (C) 2007 American Institute of Physics. [DOI: 10.1063/1.2822410]
\end{abstract}

Transflective liquid crystal displays (TR-LCDs) have been widely used in mobile devices, e.g., mobile phones, digital cameras, and personal digital assistants, because of their advantages in sunlight readability and low power consumption. ${ }^{1,2}$ A commonly employed TR-LCD consists of transmissive $(T)$ and reflective $(R)$ subpixels. The $T$ mode uses backlight while $R$ mode uses ambient light to readout the displayed images. In the $T$ mode, the backlight passes the liquid crystal (LC) medium once while the ambient light traverses the LC layer twice. To compensate for this optical path length difference, both dual cell gaps and single cell gap approaches have been developed. ${ }^{3}$ On the LC configurations, single-domain and multiple-domain planars, twisted nematic, vertical alignment (VA), in-plane switching (IPS), and fringe field switching (FFS) have been explored. ${ }^{3}$ Among them, multidomain VA, IPS, and FFS are known to exhibit a superb viewing angle. ${ }^{4}$ However, the demand in viewing angle for a hand-held LCD is less stringent than a large screen LCD television because most of the time it is viewed only by one person. For mobile displays, thin profile and light weight are highly desirable. The single-domain FFS mode offers an adequate viewing angle without using any compensation films ${ }^{5}$ and, therefore, it is an attractive approach for TR-LCDs.

In a TR-LCD, most displays require either a quarterwave plate $^{6-9}$ (QWP) or a patterned in-cell phase retarder ${ }^{10-13}$ in order to obtain a good dark state for the $R$ mode. The problems associated with QWP are the increased manufacturing cost and display thickness, and more seriously, the conventional QWP-based broadband circular polarizers exhibit a rather narrow viewing angle. ${ }^{9}$ Although forming patterned in-cell phase retarder in the reflective part of IPS or FFS based TR-LCD allows linear polarizers to be employed, the process for fabricating patterned in-cell phase retarder remains a technical challenge.

In this letter, we proposed a wide-view TR-LCD using a homogeneous alignment FFS in the $T$ mode and a hybrid alignment FFS in the $R$ mode. Such a TR-LCD exhibits a wide view, high transmittance, and good dark state without using any QWP or in-cell phase retarder. The device structures and performances of this TR-LCD are optimized using

\footnotetext{
${ }^{a)}$ Electronic mail: swu@ mail.ucf.edu.
}

a commercial three-dimensional (3D) LC simulator (TECHwIZ developed by Sanayi).

Figures 1(a) and 1(b) show the electrode structures and cross-section of the proposed TR-LCD, respectively. In the $T$ part, the electrode structure is the same as a conventional transmissive FFS-LCD which has a planar common electrode and slit pixel electrodes. ${ }^{14,15}$ The LC directors are aligned homogeneously with their optic axis parallel to the transmission axis of the top linear polarizer. The bottom polarizer is crossed to the top one. In the $R$ part, a reflective electrode with embossing patterns is connected to the common electrode, and the pixel electrodes above are also patterned with slits. To achieve a good dark state for the $R$ mode, a hybrid alignment LC cell with a phase retardation value $d \Delta n \sim \lambda / 4$ (where $d$ is the LC layer thickness, $\Delta n$ the LC birefringence, and $\lambda$ the wavelength) is formed by photoalignment. Its optic axis in the bottom substrate is $45^{\circ}$ with respect to the transmission axis of the top linear polarizer, as shown in Fig. 1(a). To make the LC phase retardation close to $\lambda / 4$, a thin overcoated layer whose thickness is $\sim 24 \%$ of the LC cell gap is formed on the top substrate of the $R$ region. As a result, the hybrid-aligned $\lambda / 4$ layer in the $R$ region together with the top linear polarizer functions as a circular polarizer. No additional QWP or in-cell phase re-

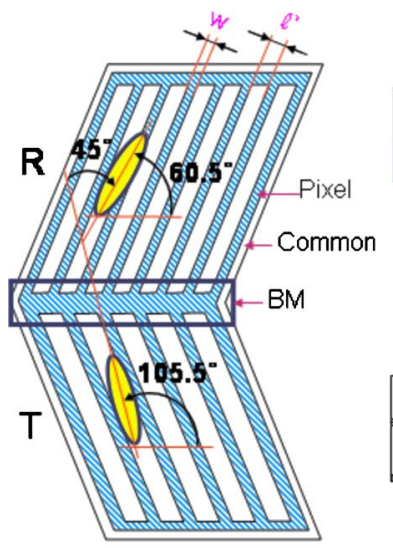

(a)

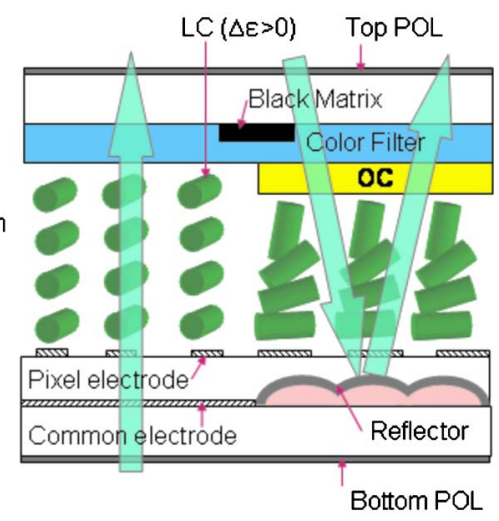

(b)
FIG. 1. (Color online) Schematic pixel structure of the FFS-based transflective LCD. BM stands for black matrix, $w$ for electrode width, and $\ell^{\prime}$ for electrode gap. 


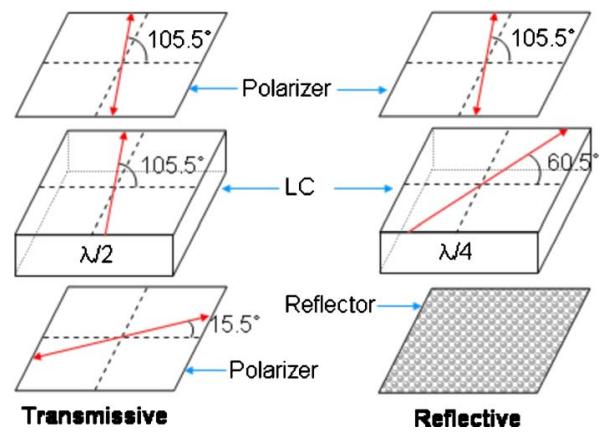

FIG. 2. (Color online) Optical configuration of the proposed transflective LCD.

tarder is required in our design. Near the wall boundaries between the homogeneous and homeotropic LC alignments, the disclination lines are present which would degrade the device contrast ratio. Therefore, this region should be blocked by a black matrix, as depicted in Fig. 1 .

Figure 2 shows the detailed optical configuration of the TR-LCD. The optic axes of the LC layer in the $T$ part and $R$ part are set parallel to and $45^{\circ}$ away from the transmission axis of the top linear polarizer, respectively. When the applied voltage is lower than a threshold $\left(V_{\mathrm{th}}\right)$, the linearly polarized light from the bottom polarizer keeps its polarization throughout the LC layer, and is blocked by the top crossed linear polarizer in the $T$ part. For the incident ambient light from the top polarizer of the $R$ part, it is first converted by the $\lambda / 4$ hybrid-aligned LC layer to a circularly polarized light, and upon reflection from the bumpy reflector it traverses the LC layer one more time and becomes linearly polarized. Its optic axis is rotated $90^{\circ}$ from the incident one and is blocked by the top linear polarizer. Under such a circumstance, both reflectance and transmittance are dark at $V=0$. This is the so-called normally black mode.

On the other hand, when a high voltage $\left(V \gg V_{\text {th }}\right)$ is applied between the pixel and common electrodes, the LC directors in the transmissive region are reoriented substantially by the fringe fields to be $\sim 45^{\circ}$ away from their initial position. This LC layer is equivalent to a half-wave plate which further rotates the incident linearly polarized light by $90^{\circ}$, making it transmit thru the top linear polarizer which results in a bright state. However, the mechanism for achieving a bright state in the reflective region is quite different. Both tilt and rotation effects of the LC directors jointly contribute to the optimal reflectance.

To calculate the electro-optic characteristics of this TR-LCD, we optimized the LC retardation value in order to maximize the contrast ratio at normal incidence. A 3D LC director simulation was performed using the commercial software TECHWIZ and the optical calculation was based on the extended $2 \times 2$ Jones matrix methods. ${ }^{16,17}$ Here, the LC material employed is M06-228 (Merck) with its physical properties listed as follows: extraordinary and ordinary refractive indices $n_{e}=1.5861$ and $n_{o}=1.4815$ (at $\lambda=589 \mathrm{~nm}$ ), dielectric anisotropy $\Delta \varepsilon=9.3$, rotational viscosity $\gamma_{1}=86 \mathrm{mPa} \mathrm{s}$, and elastic constants $K_{11}=13.3 \mathrm{pN}$, $K_{22}=7 \mathrm{pN}$, and $K_{33}=12.6 \mathrm{pN}$. The boundary conditions are as follows: in the $T$ part the surface tilt angle is $2^{\circ}$ and initial rubbing angle is $105.5^{\circ}$ from the horizontal axis which is $7^{\circ}$ from the pixel electrode stripes, as depicted in Fig. 1(a). In the $R$ part, the surface pretilt angle on the TFT array sub-

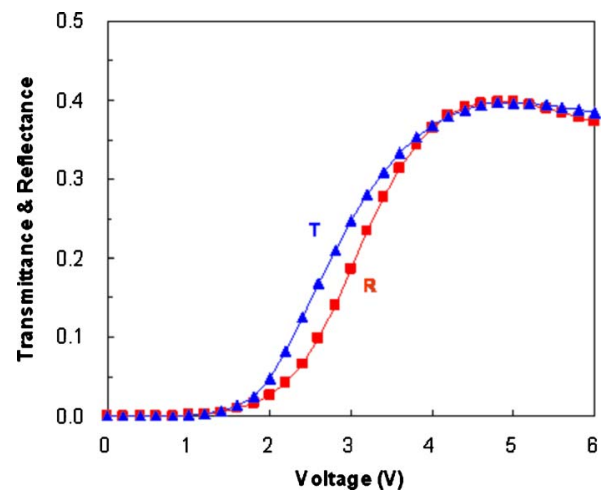

FIG. 3. (Color online) Voltage-dependent transmittance and reflectance curves of the transflective LCD.

strate is $2^{\circ}$ and initial rubbing angle is $60.5^{\circ}$ from the horizontal axis which is $7^{\circ}$ from the pixel electrode stripes, as depicted in Fig. 1(a). Besides, the pretilt angle of the LC directors on the top color filter substrate is $90^{\circ}$.

In a TR-LCD, the $T$ part has priority over the $R$ part because the latter plays an important role only under strong ambient. To reduce power consumption, the threshold voltage $V_{\text {th }}$ and bright state voltage $V_{\text {on }}$ of this display should be minimized. The $V_{\text {th }}$ of a FFS cell mainly depends on the $\Delta \varepsilon$ of the LC, electrode width $(w)$ and space $\left(\ell^{\prime}\right)$, cell gap $(d)$, and the initial LC director angle. ${ }^{5,14,18}$ Based on our calculations, we found that the optimal design of the transmissive pixel electrodes is $w=3 \mu \mathrm{m}, \ell^{\prime}=5 \mu \mathrm{m}$, and $d \sim 3.62 \mu \mathrm{m}$ for the material employed. Under such conditions, we find $V_{\text {th }} \sim 1.5 V_{\text {rms }}, V_{\text {on }} \sim 4.8 V_{\text {rms }}$, and $T_{\max } \sim 80 \%$ (normalized to the maximum transmittance of two parallel linear polarizers).

For the reflective mode, we first calculated the $d \Delta n$-dependent contrast ratio and reflectance. To find the intrinsic contrast ratio, during simulations all the interface reflections are ignored and a single wavelength $(\lambda=550 \mathrm{~nm})$ is employed. If surface reflections and bandwidth of the incident light are taken into consideration, the contrast ratio will be degraded. For $d \Delta n \sim 287 \mathrm{~nm}$, the effective phase retardation of the hybrid alignment $\mathrm{LC}$ cell is $\sim \lambda / 4$ and the maximum contrast ratio reaches 2500:1 for the reflective mode. Furthermore, to achieve a good grayscale match with the transmissive mode, we optimized the $\left(w, \ell^{\prime}\right)$ of the pixel electrode of the reflective part FFS structure by calculating its reflectance and operation voltage based on $d \Delta n$ $\sim 287 \mathrm{~nm}$. In particular, we varied the $\left(w, \ell^{\prime}\right)$ of the reflective pixel electrode from $[2 \mu \mathrm{m}, 2 \mu \mathrm{m}]$ to $[6 \mu \mathrm{m}, 6 \mu \mathrm{m}]$ and found that the optimal $\left[w, \ell^{\prime}\right]$ value is $[2 \mu \mathrm{m}, 4 \mu \mathrm{m}]$. Under such a circumstance, the reflectance is high and the voltage-dependent transmittance (VT) and reflectance (VR) curves match well to enable a single gamma driving.

Figure 3 shows the simulated VT and VR curves of the proposed cell structure using the optimized cell parameters, namely, the cell gaps of $T$ and $R$ parts are 3.62 and $2.73 \mu \mathrm{m}$, and $\left[w, \ell^{\prime}\right]$ of the $T$ and $R$ pixel electrodes are [3 $\left.\mu \mathrm{m}, 5 \mu \mathrm{m}\right]$ and $[2 \mu \mathrm{m}, 4 \mu \mathrm{m}]$, respectively. In the full bright state, the reflectance is over 0.4 , i.e., $80 \%$ of the maximum reflectance. And a reasonably good grayscale match between the $T$ and $R$ modes is obtained which allows the driving of both $T$ and $R$ modes with a single gamma curve.

Figure 4 shows the isocontrast contour plots of the $T$ and $R$ modes. Without using any compensation films, the $T$ mode shows 10:1 contrast ratio without grayscale inversion over 

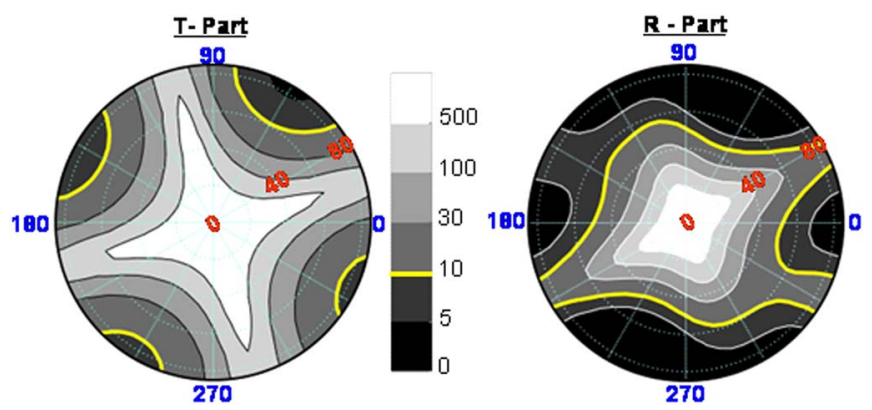

FIG. 4. (Color online) Isocontrast contour plots for the transmissive and reflective parts of the transflective LCD.

$60^{\circ}$ viewing cone and $R$ mode over $50^{\circ}$. These viewing angles are adequate for mobile displays using a small-sized LCD.

In conclusion, we have proposed a FFS-based transflective LCD that fuses a homogenous alignment in the $T$ part and a hybrid one in the $\mathrm{R}$ part. Our results show that this transflective display exhibits a high transmittance and reflectance, well matched VT and VR curves, simple device structure, and wide viewing angle. This device is particularly attractive for mobile displays that require low power consumption, high image quality, and wide viewing angle.

The authors are indebted to Chi-Mei Optoelectronic Corporation (CMO), Taiwan, for the financial support.
${ }^{1}$ M. Kubo, S. Fujioka, T. Ochi, Y. Narutaki, T. Shinomiya, Y. Ishii, and F. Funada, Proceedings of the Sixth International Display Workshops, 1999 (unpublished), p. 183.

${ }^{2}$ H. Baek, Y. B. Kim, K. S. Ha, D. G. Kim, and S. B. Kwon, Proceedings of the Seventh International Display Workshops, 2000 (unpublished), p. 41. ${ }^{3}$ For a review, see X. Zhu, Z. Ge, T. X. Wu, and S. T. Wu, J. Disp. Technol. 1, 15 (2005).

${ }^{4}$ For a review, see R. Lu, X. Zhu, S. T. Wu, Q. Hong, and T. X. Wu, J. Disp. Technol. 1, 1 (2005).

${ }^{5}$ S. H. Lee, S. L. Lee, and H. Y. Kim, Appl. Phys. Lett. 73, 2881 (1998).

${ }^{6}$ S. T. Wu and C. S. Wu, Appl. Phys. Lett. 68, 1455 (1996).

${ }^{7}$ C. J. Yu, J. Kim, D. W. Kim, and S. D. Lee, SID Int. Symp. Digest Tech. Papers 35, 642 (2004).

${ }^{8}$ Z. Ge, X. Zhu, R. Lu, T. X. Wu, and S. T. Wu, Appl. Phys. Lett. 90, 221111 (2007).

${ }^{9}$ Q. Hong, T. X. Wu, R. Lu, and S. T. Wu, Opt. Express 13, 10777 (2005). ${ }^{10}$ J. H. Song, Y. J. Lim, M. H. Lee, S. T. Shin, and S. H. Lee, Appl. Phys. Lett. 87, 011108 (2005).

${ }^{11}$ J. B. Park, H. Y. Kim, Y. H. Jeong, S. Y. Kim, and Y. J. Lim, Jpn. J. Appl. Phys., Part 1 44, 7524 (2005).

${ }^{12}$ S. Hirota, S. Oka, and O. Itou, SID Int. Symp. Digest Tech. Papers 38, 1661 (2007).

${ }^{13}$ H. Imayama, J. Tanno, K. Igeta, M. Morimoto, S. Komura, T. Nagata, O. Itou, and S. Hirota, SID Int. Symp. Digest Tech. Papers 38, 1651 (2007).

${ }^{14}$ S. H. Hong, I. C. Park, H. Y. Kim, and S. H. Lee, Jpn. J. Appl. Phys., Part 2 39, L527 (2000).

${ }^{15}$ H. Y. Kim, G. R. Jeon, D. S. Seo, M. H. Lee, and S. H. Lee, Jpn. J. Appl. Phys., Part 1 41, 2944 (2001).

${ }^{16}$ A. Lien, Appl. Phys. Lett. 57, 2767 (1990).

${ }^{17}$ Z. Ge, X. Zhu, T. X. Wu, and S. T. Wu, J. Opt. Soc. Am. A 22, 966 (2005).

${ }^{18}$ H. Y. Kim, S. H. Hong, J. M. Rhee, and S. H. Lee, Liq. Cryst. 30, 1285 (2003). 